\title{
Silencing of aquaporin 5 inhibits the growth of A549 lung cancer cells in vitro and in vivo
}

\author{
LIN ZHANG $^{1,2}$, JIA LU ${ }^{1,2}$, HONGYAN ZHOU $^{2}$, ZHENWU DU $^{1}$ and GUIZHEN ZHANG ${ }^{1}$ \\ ${ }^{1}$ Central Laboratory, The Second Hospital of Jilin University, Changchun, Jilin 130041; ${ }^{2}$ Department of Ophthalmology, \\ China-Japan Union Hospital, Jilin University, Changchun, Jilin 130033, P.R. China
}

Received November 22, 2017; Accepted March 1, 2018

DOI: $10.3892 /$ ijo.2018.4326

\begin{abstract}
The water channel protein aquaporin 5 (AQP5) is highly expressed in numerous tumors. However, its expression pattern and functions in lung cancer in humans remain unknown. In the present study, the role of AQP5 in the development of lung malignancies was examined. A short hairpin RNA construct targeting AQP5 mRNA was transfected into A549 cells to generate a lung cancer cell line in which AQP5 expression was stably silenced. In vitro and in vivo experiments were then performed to establish the effects of AQP5 on A549 cell apoptosis, proliferation and cell cycle progression. The results demonstrated that AQP5 silencing significantly inhibited the proliferation and promoted the apoptosis of A549 lung cancer cells in vitro and in vivo. In addition, it resulted in decreased activation of the extracellular signal-regulated kinase 1/2 signaling pathway in A549 cells, and reduced levels of the downstream proteins c-Fos and phosphorylated cAMP response element-binding protein. Furthermore, inhibition of AQP5 expression effectively reduced the tumorigenicity of A549 cells in vivo. In conclusion, silencing of AQP5 suppressed the growth of A549 cells in vitro and in vivo, suggesting that it may serve as a therapeutic target in lung cancer.
\end{abstract}

\section{Introduction}

In recent years, lung cancer has been ranked among the primary malignant tumors associated with the highest morbidity and mortality worldwide (1-3). Non-small-cell lung cancer (NSCLC) is the most common type of this disease, accounting for $\sim 80 \%$ of all lung malignancies $(4,5)$. Treatment regimens vary and may include radiotherapy, chemotherapy, molecular targeted therapies and surgery, alone or in combination. However, the 5-year survival rate

Correspondence to: Professor Guizhen Zhang, Central Laboratory, The Second Hospital of Jilin University, 18 Ziqiang Street, Changchun, Jilin 130041, P.R. China

E-mail: zhangguizhenjlu@163.com

Key words: lung cancer, aquaporin 5, proliferation, apoptosis, extracellular signal-regulated kinase signaling pathway among NSCLC patients remains poor $(6,7)$. Recent technological advances have allowed researchers to identify genetic factors that play essential roles in the pathophysiology of NSCLC, providing novel approaches for the development of treatments for this disease $(8,9)$. Therefore, elucidation of the molecular mechanisms underlying NSCLC and identification of new genetic targets for its treatment should be urgently conducted.

Aquaporins (AQPs), a family of small (30 kDa/monomer) transmembrane water channel proteins, were first described in the late 1950s in red blood cells and later in renal epithelia, and have been identified as key potential targets for novel antitumor therapies (10-12). AQP5, one of the most important members of this protein family, is expressed in virtually all normal tissues and contributes to cellular regulation of water homeostasis $(13,14)$. Malignant tumor cells have been reported to take advantage of this process to facilitate their proliferation and development $(15,16)$. In addition, AQP5 gene silencing has been reported to inhibit such proliferation, suggesting that altered AQP5 expression is crucial in tumor progression $(17,18)$. Numerous studies have demonstrated that AQP5 is upregulated in NSCLC, and that AQP5-overexpressing tumor cells are associated with increased tumor growth rates and progression owing to heightened proliferation (19-21). However, the association between the AQP5 gene and NSCLC is yet to be fully elucidated.

The aims of the present study were to establish whether AQP5 serves an important role in NSCLC and how AQP5 gene silencing may influence cell proliferation and apoptosis in this malignancy. Furthermore, the underlying molecular mechanisms were examined to provide a new direction for the treatment of NSCLC.

\section{Materials and methods}

Cell culture. The cell lines A549, H358, HCC827 and H1299 were obtained from the Cell Bank of the Type Culture Collection of the Chinese Academy of Sciences (Shanghai, China). The MRC-5 cell line was also purchased from Guangzhou Jinnio Biotechnology Co., Ltd. (Guangzhou, China). All cells were cultured in Dulbecco's modified Eagle's medium (DMEM; Gibco; Thermo Fisher Scientific, Inc., Waltham, MA, USA) supplemented with $10 \%$ fetal bovine serum (FBS; Gibco; Thermo Fisher Scientific, Inc.) in a humidified atmosphere 
containing $5 \% \mathrm{CO}_{2}$ at $37^{\circ} \mathrm{C}$. Cells in the exponential phase of growth were used in subsequent experiments.

Generation of an AQP5-silenced lung cancer cell line. The AQP5 short hairpin RNA (shRNA) expression construct, namely AQP5-pGCH1/Neo, and the non-targeting control construct, namely NC-pGCH1/Neo, were designed and synthesized by Sangon Biotech Co., Ltd. (Shanghai, China). The sequences of the AQP5 and NC shRNA molecules were 5'-CCGGCCATCATCAAAGGCACGTATGCTCGAGCATA CGTGCCTTTGATGATGGTTTTTG-3' and 5'-GATCCCACT ACCGTTGTTATAGGTGTTCAAGAGACACCTATAACAA CGGTAGTTTTTTTCCAAA-3', respectively. A549 cells, in which AQP5 was found to be highly expressed (Fig. 1A and B), were transfected with AQP5-pGCH1/Neo or NC-pGCH1/Neo using Lipofectamine ${ }^{\circledR} 2000$ (Invitrogen; Thermo Fisher Scientific, Inc.) according to the manufacturer's protocol. At $24 \mathrm{~h}$ after transfection, the cells were exposed to $200 \mu \mathrm{g} / \mathrm{ml}$ G418 (Invitrogen; Thermo Fisher Scientific, Inc.) for over a week in order to select clones stably expressing the constructs. Positive clones were selected for further identification.

Reverse transcription-quantitative polymerase chain reaction $(R T-q P C R)$. Total RNA was extracted from cultured cells with TRIzol reagent (Tiangen Biotech Co., Ltd., Beijing, China) according to the manufacturer's protocol, and cDNA was then synthesized by reverse transcription. The total PCR reaction volume was $20 \mu \mathrm{l}$ and consisted of $1 \mu \mathrm{l}$ of cDNA, $0.5 \mu \mathrm{l}$ of each primer, $10 \mu \mathrm{l}$ of SYBR-Green master mix, and $8 \mu \mathrm{l}$ of $\mathrm{ddH}_{2} \mathrm{O}$. The PCR reaction program was as follows: $95^{\circ} \mathrm{C}$ for $10 \mathrm{~min}$; 40 cycles of $95^{\circ} \mathrm{C}$ for $10 \mathrm{sec}, 60^{\circ} \mathrm{C}$ for $20 \mathrm{sec}, 72^{\circ} \mathrm{C}$ for $30 \mathrm{sec}$; and $4^{\circ} \mathrm{C}$ for $5 \mathrm{~min}$. The primers used to amplify AQP5 and $\beta$-actin cDNA were as follows: AQP5 sense, 5'-CTATGAG TCCGAGGAGGATT-3', and antisense, 5'-GCTTCGCTGTC ATCTGTT-3'; $\beta$-actin sense, 5'-CCTGTACGCCAACACAG TGC-3', and antisense, 5'-ATACTCCTGCTTGCTGATCC-3'. the relative mRNA levels in each sample were calculated using the $2^{-\Delta \Delta C q}$ method (22), using $\beta$-actin as a reference. $\mathrm{qPCR}$ was performed using SYBR-Green Master Mix (Tiangen Biotech Co., Ltd.) in an Exicycler ${ }^{\mathrm{TM}} 96$ quantitative fluorescence analyzer (Bioneer Corporation, Daejeon, Korea).

Western blotting. Cells were lysed (Beyotime Institute of Biotechnology, Haimen, China), and the concentration of protein in lysates was determined by bicinchoninic acid assays (Beyotime). Total proteins $(40 \mu \mathrm{g})$ from each sample were separated by sodium dodecyl sulfate-polyacrylamide gel electrophoresis (5\% acrylamide and 10\% separating gel) and then electroblotted onto a polyvinylidene fluoride membrane (EMD Millipore, Bedford, MA, USA). Subsequent to blocking with evaporated milk $37^{\circ} \mathrm{C}$, the membrane was incubated at $4^{\circ} \mathrm{C}$ overnight with primary antibodies against the following: AQP5, cleaved caspase-3, B-cell lymphoma 2 (Bcl-2), Bcl-2-associated $\mathrm{X}$ protein (Bax), extracellular signalregulated kinase (ERK), phosphorylated (p)-ERK, c-Fos, or p-cAMP response element-binding protein (p-CREB) (all diluted 1:1,000; Abcam, Cambridge, MA, USA). Next, the membranes were exposed to a horseradish peroxidase-labeled secondary antibody (1:5,000; Abcam) at room temperature for $45 \mathrm{~min}$. Proteins were visualized using an enhanced chemiluminescence reagent (Beyotime), and band intensities were analyzed with Gel-Pro Analyzer software version 4 (Media Cybernetics, Inc., Rockville, MD, USA) for comparison of the gray densities.

Colony formation assay. Cells were seeded onto $35-\mathrm{mm}$ dishes at a density of 200 cells per dish and incubated for 14 days at $37^{\circ} \mathrm{C}$ in a humidified atmosphere containing $5 \% \mathrm{CO}_{2}$. The resulting colonies were rinsed with PBS, at room temperature and fixed with paraformaldehyde for $20 \mathrm{~min}$, and then stained with Giemsa solution (Nanjing Jiancheng Bioengineering Institute, Nanjing, China) for 5-8 min. The number of colonies consisting of at least 50 cells was then counted under a microscope. Colony formation efficiency was calculated as follows: (Number of colonies / number of inoculated cells) x 100\%.

3-(4,5-Dimethylthiazol-2-yl)-2,5-diphenyltetrazolium bromide (MTT) assay. Cells were seeded into 96-well microplates at a density of $2 \times 10^{3}$ cells/well and with each well containing $200 \mu 1$ DMEM supplemented with 10\% FBS. The cells were allowed to adhere prior to the addition of MTT (Sigma-Aldrich; Merck KGaA, Darmstadt, Germany) to each well at a final concentration of $0.2 \mathrm{mg} / \mathrm{ml}$ at various time-points $(12,24,48,72$ and $96 \mathrm{~h}$ ). Subsequent to incubation at $37^{\circ} \mathrm{C}$ for $4 \mathrm{~h}$, the optical density at $490 \mathrm{~nm}$ was measured using an ELx800 microplate reader (BioTek Instruments, Inc., Winooski, VT, USA).

Flow cytometry for cell cycle progression and apoptosis analyses. For cell cycle analysis, cells were fixed in $70 \%$ cold ethanol at $4^{\circ} \mathrm{C}$ for $3 \mathrm{~h}$, washed in PBS, and then resuspended in staining buffer containing $25 \mu \mathrm{l}$ propidium iodide (PI) and $10 \mu 1 \mathrm{RNaseA}$ (Abcam). The cell suspension was incubated for $45 \mathrm{~min}$ in the dark at $37^{\circ} \mathrm{C}$, and then subjected to cell cycle distribution analysis by flow cytometry using a FACSCalibur instrument (BD Biosciences, Franklin Lakes, NJ, USA).

In addition, apoptotic cells were identified using fluorescence-activated cell sorting and an apoptosis detection kit (Beyotime Institute of Biotechnology, Nanjing, China), according to the manufacturer's protocol. Cells were harvested, centrifuged at $4^{\circ} \mathrm{C}, 503 \mathrm{xg}, 5 \mathrm{~min}$, and then washed with PBS prior to resuspending in $400 \mu \mathrm{l}$ binding buffer. Next, $5 \mu \mathrm{l}$ Annexin V-fluorescein isothiocyanate was added to the cell suspensions, which were then incubated at $2-8^{\circ} \mathrm{C}$ for $15 \mathrm{~min}$ in the dark. PI $(10 \mu \mathrm{l})$ was subsequently added, and the cell suspensions were incubated at $28^{\circ} \mathrm{C}$ for $5 \mathrm{~min}$ in the dark. The cells were then analyzed by flow cytometry within $1 \mathrm{~h}$ of this treatment.

In vivo experiments. A total of $18 \mathrm{BALB} / \mathrm{c}$ nude mice (4-6-week-old; weight, $20 \mathrm{~g}$ ) were purchased from Beijing Vital River Laboratory Animal Technology Co., Ltd. (Beijing, China). The animals were maintained under pathogen-free conditions at $22^{\circ} \mathrm{C}$ and $40-50 \%$ humidity with a $12 / 12$-h light/ dark cycle, and had ad libitum access to food and water. Their care and treatment and all animal experiments were approved by the Experimental Animal Ethics Committee of Jilin University (Changchun, China). Each mouse was randomly assigned to one of following three groups (6 mice in each group): A549 (untransfected cells), vector (non-targeting control construct) or AQP5 shRNA groups. Cells $\left(1 \times 10^{6}\right)$ were suspended in 

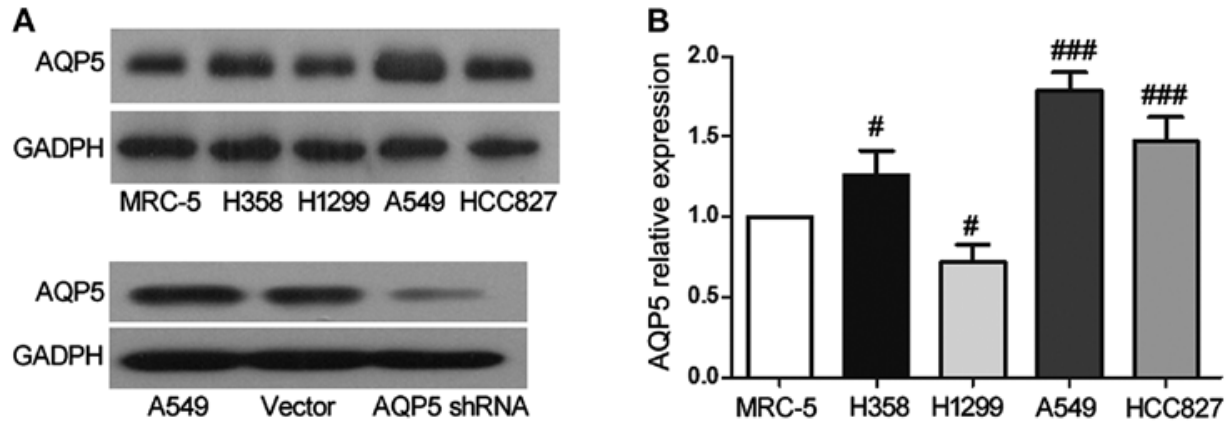

D
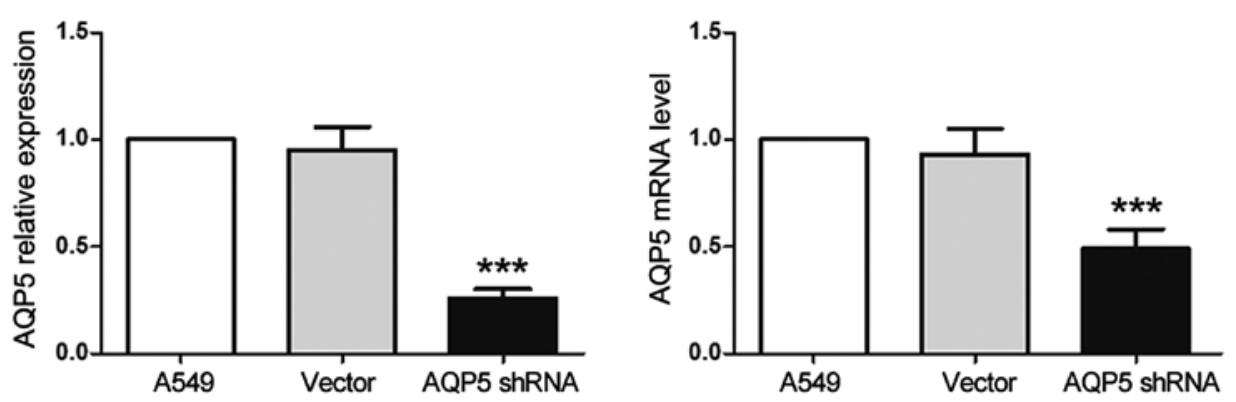

Figure 1. Stable expression of AQP5 shRNA plasmid. (A) Western blot analysis, with GADPH used as an internal control for grayscale analysis. (B) Quantified results of AQP5 protein expression levels in multiple human lung cancer cell lines and in human lung fibroblast MRC-5 cell line. (C) Protein expression levels in cells stably transfected with non-targeting control (vector) or AQP5 shRNA constructs. (D) Reverse transcription-quantitative polymerase chain reaction analysis was conducted to measure AQP5 expression in stably transfected cells. Data representative of three independent experiments are shown, and are presented as the mean \pm standard deviation. ${ }^{\#} \mathrm{P}<0.001,{ }^{\# \# *} \mathrm{P}<0.001$ vs. the MRC-5 group. ${ }^{* * * *} \mathrm{P}<0.001$, vs. the A549 control group. AQP5, aquaporin 5; shRNA, short hairpin RNA.

$0.2 \mathrm{ml}$ normal saline and inoculated subcutaneously into the right breast pads of the mice; and the A549 group with $0.2 \mathrm{ml}$ saline only. Tumor volumes in mice were determined using the following formula: Tumor volume $=\left(\mathrm{a} \mathrm{x}^{2}\right) / 2$, where $\mathrm{a}$ and $\mathrm{b}$ are the larger and smaller of the two dimensions, respectively. Mice were sacrificed after 30 days, and tumor tissues were removed and fixed in polyformaldehyde after imaging of the tumors for further examination.

Detection of apoptosis using terminal deoxynucleotidyl transferase dUTP nick end labeling (TUNEL) assay. Apoptosis in the xenograft tumor tissue was detected using an In Situ Cell Death Detection kit (Roche Diagnostics GmbH, Mannheim, Germany) according to the manufacturer's protocol. Briefly, paraffin sections $\left(1 \mathrm{~cm}^{2}\right)$ were treated with a solution of $\mathrm{H}_{2} \mathrm{O}_{2}$ in order to inactivate endogenous peroxidases, and incubated with $50 \mu 1 \mathrm{TUNEL}$ reaction solution at $37^{\circ} \mathrm{C}$ for $60 \mathrm{~min}$. Subsequently, the sections were washed with PBS and incubated with $50 \mu \mathrm{l}$ Converter-POD working solution at $37^{\circ} \mathrm{C}$ for $30 \mathrm{~min}$. Following development using 3,3'-diaminobenzidine and staining of nuclei with hematoxylin, the sections were observed under a microscope and images were captured.

Statistical analysis. Data are presented as the mean \pm standard deviation. Comparisons between groups were performed using one-way analysis of variance, and multiple comparisons were performed using the Bonferroni post hoc test. GraphPad Prism version 5.0 software (GraphPad Software, Inc., La Jolla, CA, USA) was used to analyze data and generate graphs. Differences associated with P-values of $<0.05$ were considered as statistically significant.

\section{Results}

AQP5 silencing by stable expression of shRNA. In order to examine the function of AQP5 in lung cancer cells, western blotting was used to measure its expression in various human lung cancer cell lines and lung fibroblast cells. Of the cell lines tested, AQP5 expression was observed in all the experimental cell lines, with the highest expression observed in the A549 cells compared with the MRC-5 cell line (Fig. 1A and B). Western blotting also revealed that transfection of the A549 cells with AQP5 shRNA resulted in the significant inhibition of AQP5 protein expression to only $26 \%$ of that in the A549 (untransfected) group (Fig. 1A and $\mathrm{C} ; \mathrm{P}<0.001$ ). These results were further confirmed by RT-qPCR, which indicated that the expression of AQP5 mRNA was also significantly inhibited in the AQP5 shRNA group and was only $49 \%$ of that in the A549 group (Fig. 1D; $\mathrm{P}<0.001$ ).

AQP5 downregulation inhibits A549 cell proliferation and cell cycle progression. A colony formation assay was used to investigate the possible role of AQP5 in A549 cell growth. The clonogenicity of AQP5 shRNA cells was found to be significantly reduced compared with that of the A549 and vector groups (Fig. $2 \mathrm{~A}$ and $\mathrm{B} ; \mathrm{P}<0.01$ ). Furthermore, the MTT assay demonstrated that proliferation of AQP5 shRNA cells at $72 \mathrm{~h}(\mathrm{P}<0.05)$ and $96 \mathrm{~h}(\mathrm{P}<0.01)$ of culture was markedly lower compared with that of cells in the A549 group (Fig. 2C).

To further explore the effect of AQP5 silencing on cell cycle progression, flow cytometry was used to evaluate the distribution of cells in each phase of the cell cycle (Fig. 2D and E). In the AQP5 shRNA group, numerous cells were in 
A

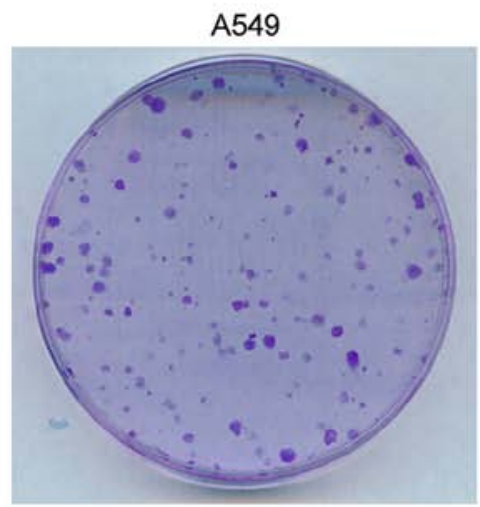

B

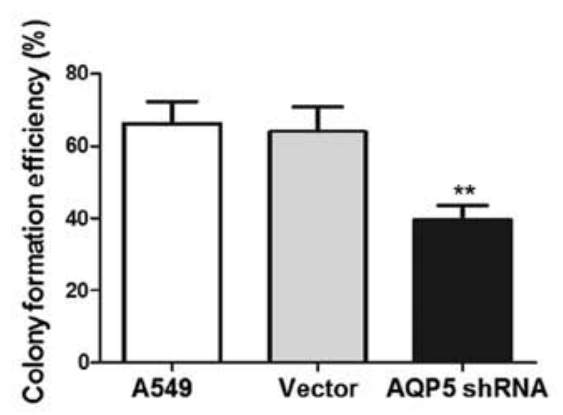

E

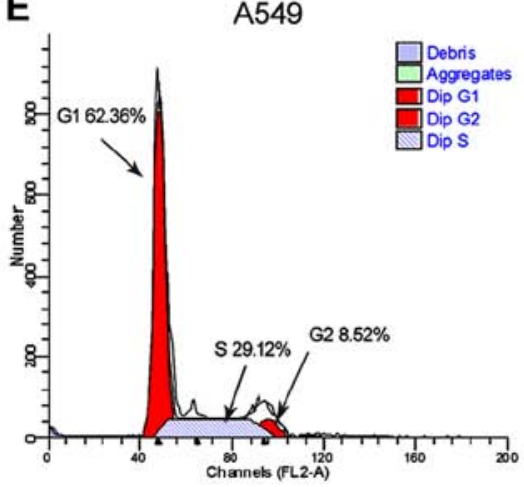

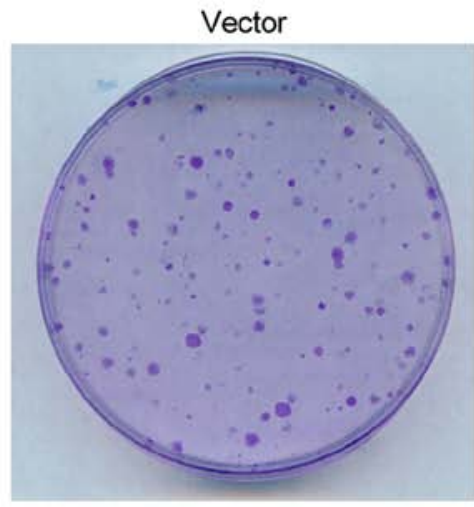

C
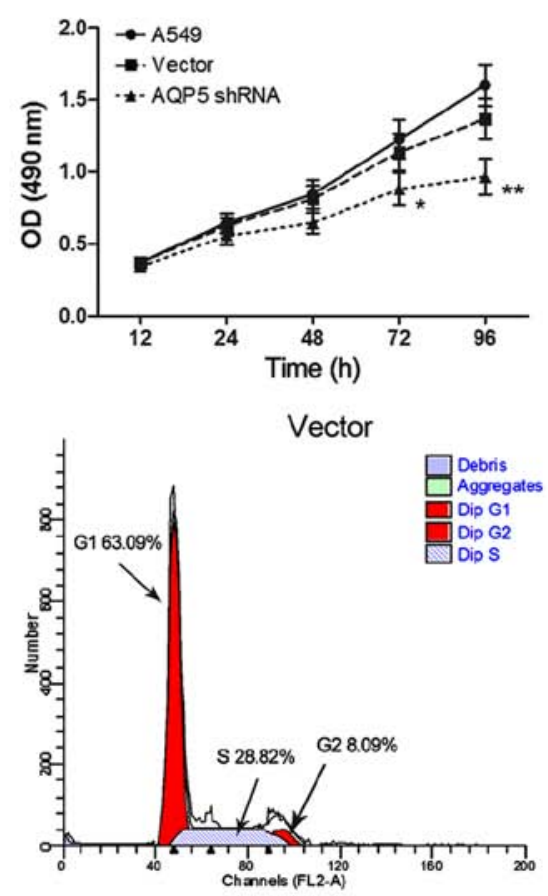

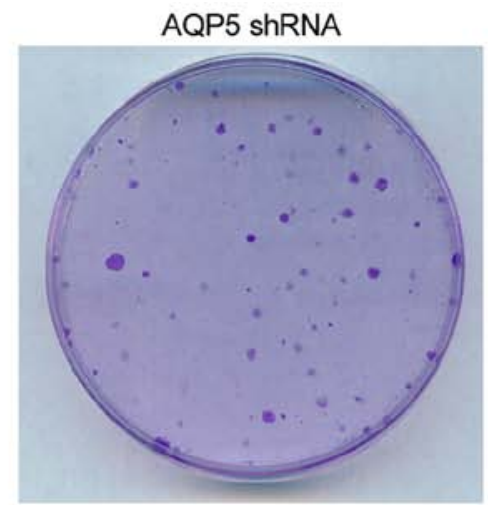

D

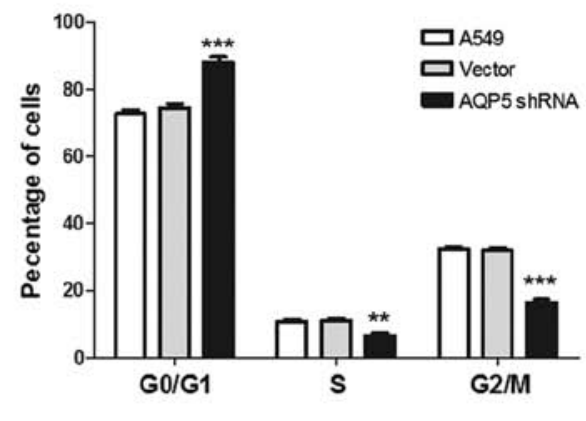

AQP5 shRNA

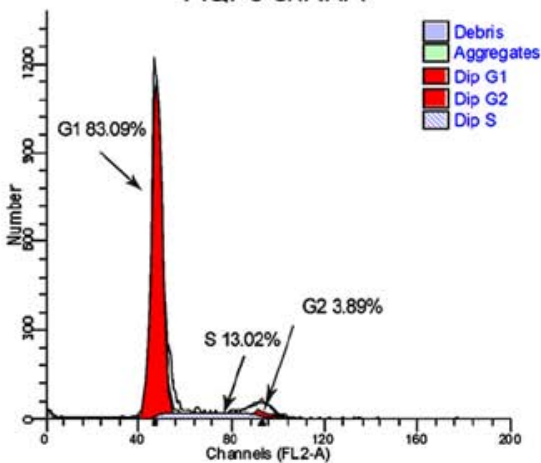

Figure 2. AQP5 downregulation inhibits A549 cell proliferation and cell cycle progression. (A) Colony formation assay was conducted to evaluate the tumorigenicity of AQP5-silenced cells. (B) Colony formation efficiency is shown. (C) An MTT assay was performed to assess cell proliferation in vitro. (D) Percentage of cells in each cell cycle phase and (E) flow cytometry results are presented. Cultured cells were fixed, stained with PI, and subjected to flow cytometry for cell cycle analysis. Cells in the lower right quadrant were considered to be early apoptotic cells and were statistically analyzed. Data representative of three experiments are shown, and data are presented as the mean \pm standard deviation. ${ }^{*} \mathrm{P}<0.05,{ }^{* *} \mathrm{P}<0.01$ and ${ }^{* * *} \mathrm{P}<0.001$, vs. the A549 control group. AQP5, aquaporin 5; shRNA, short hairpin RNA; OD, optical density.

G0/G1 phase $(\mathrm{P}<0.001)$, whereas the number of those in $\mathrm{S}$ $(\mathrm{P}<0.01)$ and $\mathrm{G} 2 / \mathrm{M}(\mathrm{P}<0.001)$ phases was significantly reduced as compared with the A549 control group. No evident difference in cell cycle distribution was noted between the A549 and vector groups.

AQP5 downregulation induces A549 cell apoptosis. Flow cytometry was employed to establish whether AQP5 downregulation significantly affected A549 cell apoptosis. The apoptotic ratio in the AQP5 shRNA group was observed to be 1.54-fold higher compared with that in the A549 group (Fig. 3A and B; $\mathrm{P}<0.001$ ); thus, apoptosis of A549 cells was significantly increased by AQP5 silencing. Western blotting was subsequently used to measure the expression levels of the apoptosis suppressor Bcl-2 and the downstream executioners of apoptosis, Bax and cleaved caspase-3. The expression levels of cleaved caspase-3 and Bax in cells of the AQP5 shRNA group was 2.68-fold (Fig. 3C and D; $\mathrm{P}<0.001$ ) and 2.31-fold (Fig. 3C and $\mathrm{E} ; \mathrm{P}<0.001$ ) higher, respectively, when compared with those in cells of the A549 group. In addition, the expression of Bcl-2 in the AQP5 shRNA group was only $56 \%$ of that in the A549 group (Fig. 3C and F; $\mathrm{P}<0.001$ ). These findings further indicated that downregulation of AQP5 was able to induce A549 cell apoptosis.

AQP5 downregulation inhibits ERK signaling. The mechanism by which AQP5 silencing affects A549 cell behavior was investigated using western blotting. It was observed that the protein levels of p-ERK, c-Fos and p-CREB in the AQP5 shRNA group were 31\% $(\mathrm{P}<0.001), 41 \%(\mathrm{P}<0.001)$ and $53 \%(\mathrm{P}<0.001)$ of those in the A549 group, respectively (Fig. 4). These findings indicated that the downregulation of 
A

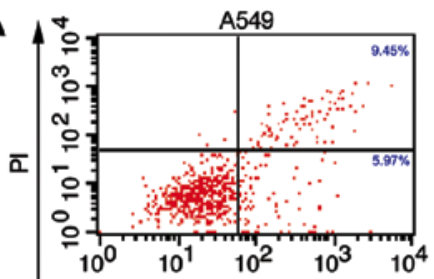

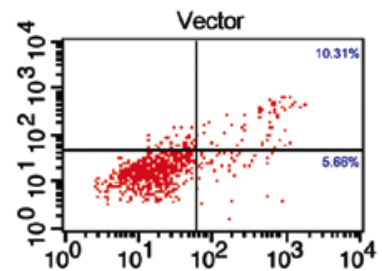

Annexin V-FITC
B

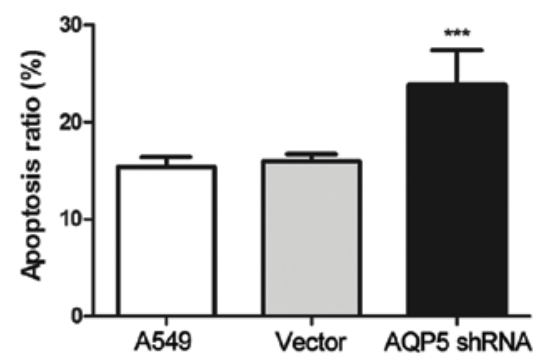

C

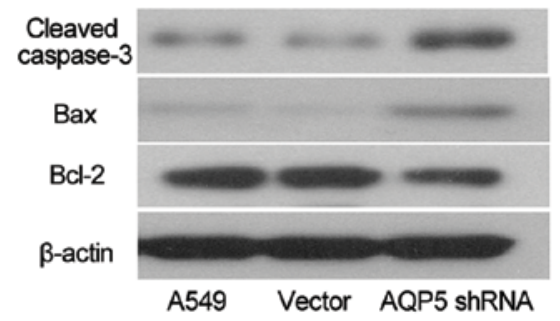

$\mathbf{F}$

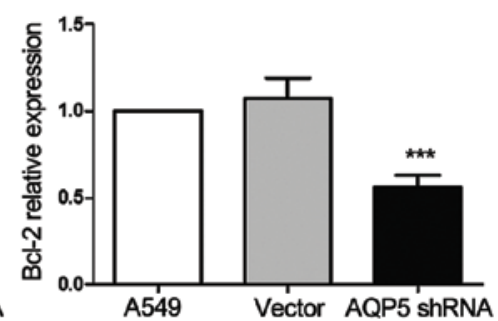

Figure 3. AQP5 downregulation induces apoptosis of A549 cells. (A) Flow cytometry results and (B) apoptotic ratio of cells are presented. The apoptosis of cultured cells was tested by double-staining with FITC-conjugated Annexin V and PI, followed by flow cytometry analysis. (C) Western blot analysis results, with $\beta$-actin used as an internal control for grayscale analysis. (D) Cleaved caspase-3, (E) Bax and (F) Bcl-2 relative protein levels in AQP5-silenced cells. Data representative of three experiments are shown, and data are presented as the mean \pm standard deviation. ${ }^{* * *} \mathrm{P}<0.001, \mathrm{vs}$. the A549 control group. AQP5, aquaporin 5; shRNA, short hairpin RNA; FITC, fluorescein isothiocyanate; Bcl-2, B-cell lymphoma 2; Bax, Bcl-2-associated X protein.

A

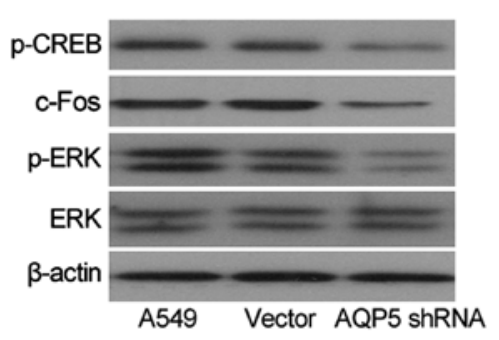

C

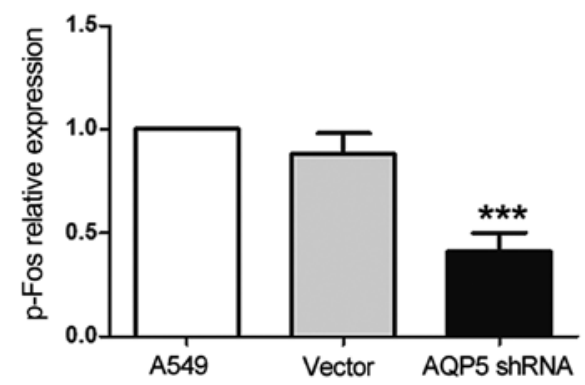

B

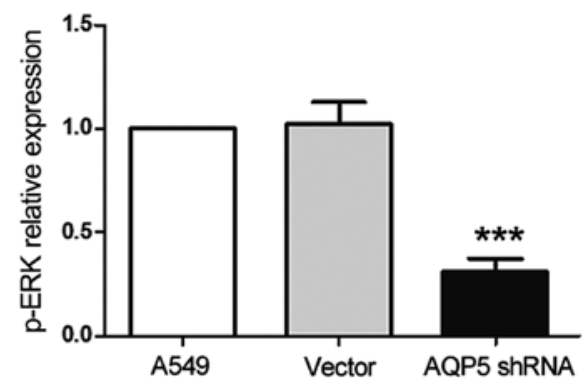

D

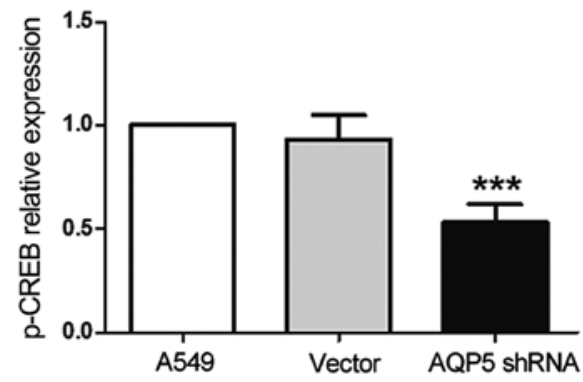

Figure 4. AQP5 downregulation inhibits the ERK signaling pathway. (A) Western blot analysis was performed to determine levels of ERK, p-ERK, c-Fos and p-CREB proteins in AQP5-silenced cells. $\beta$-actin was used as an internal control for grayscale analysis. (B) p-ERK, (C) c-Fos and (D) p-CREB protein levels are displayed. Data representative of three experiments are shown, and data are presented as the mean \pm standard deviation. ${ }^{* * *} \mathrm{P}<0.001$, vs. the A549 control group. AQP5, aquaporin 5; shRNA, short hairpin RNA; ERK, extracellular signal-regulated kinase; CREB, cAMP response element-binding protein; p-, phosphorylated. 

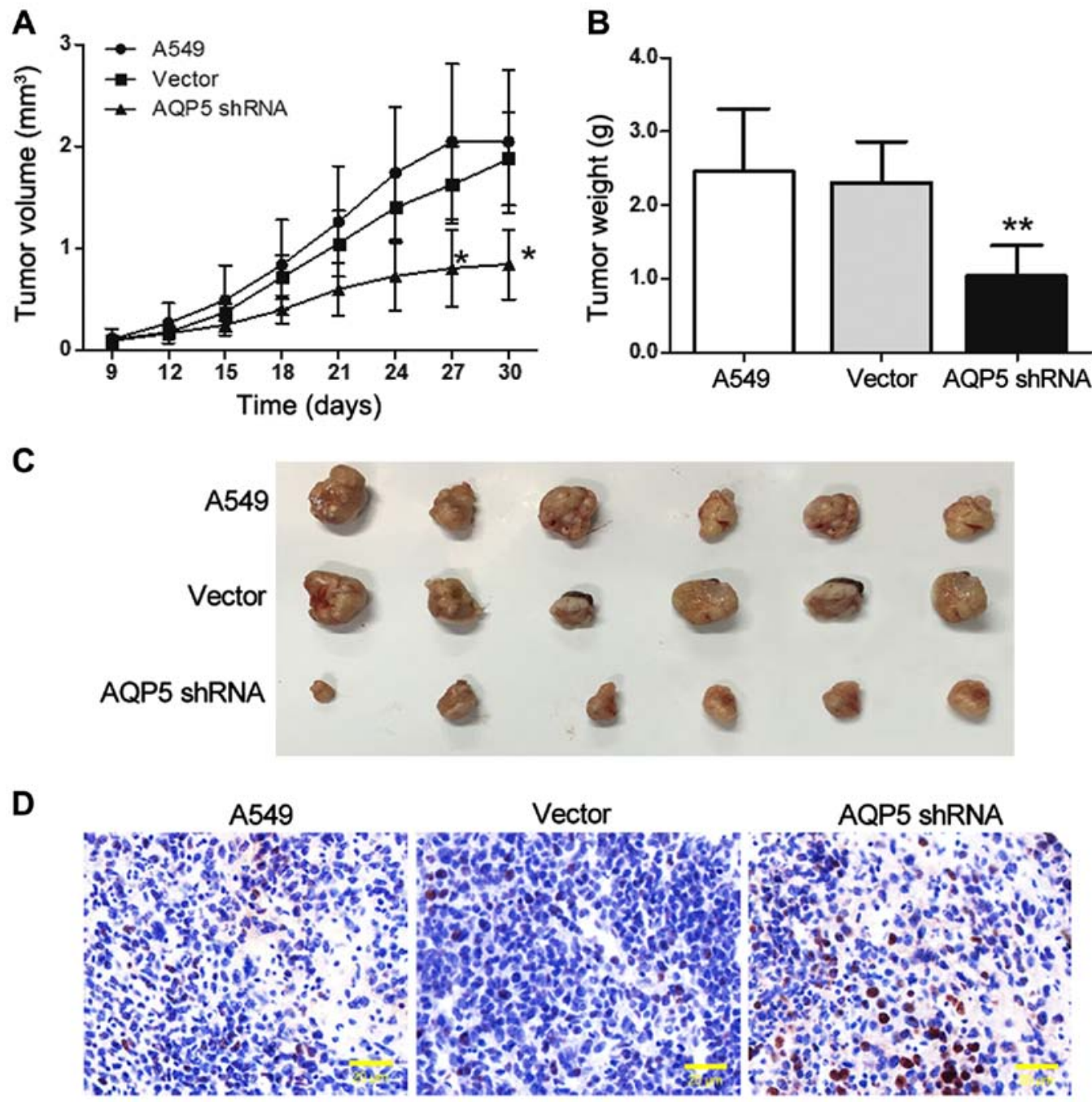

Figure 5. AQP5 downregulation inhibits A549 cell growth in vivo. Non-transfected, non-targeting shRNA-transfected or AQP5 shRNA-transfected A549 cells ( $1 \times 10^{6}$ cells each) were subcutaneously inoculated into 4-6-week-old nude mice. (A) Tumor volume assay, (B) tumor weight assay and (C) xenograft tumor images are displayed. (D) Xenograft tumor tissues were embedded in paraffin, sectioned and subjected to a terminal deoxynucleotidyl transferase dUTP nick end labeling assay to detect apoptotic cells. Data are representative of all of the animals examined. ${ }^{~} \mathrm{P}<0.05$ and ${ }^{* *} \mathrm{P}<0.01$, vs. the A549 control group. AQP5, aquaporin 5; shRNA, short hairpin RNA.

AQP5 inhibited the activation of the ERK signaling pathway by altering the phosphorylation levels of important ERK pathway proteins, Fos and CREB.

AQP5 downregulation inhibits A549 cell growth in vivo. Based on the results of the in vitro experiments, the present study next investigated the role of AQP5 in A549 cell growth in vivo. The results revealed that tumor volumes and weights in the AQP5 shRNA group were significantly lower in comparison with those in the A549 group (Fig. 5A-C). Furthermore, the TUNEL assay revealed that the number of apoptotic cells was higher in the tumor tissues of the AQP5 shRNA group as compared with those of the A549 groups (Fig. 5D). Thus, downregulation of AQP5 expression may delay lung cancer progression.

\section{Discussion}

Lung cancer remains one of the most common malignancies worldwide, and morbidity and mortality associated with this disease have been increasing (3). Despite advances in various treatment options and systemic therapy, such as chemotherapy, in combination with surgery and radiotherapy, the 5-year survival rate of lung cancer patients remains poor (6). AQP5 is one of the most important members of the AQP family, a group of water-transporting transmembrane proteins. Previous studies have demonstrated that due to the particular structure and characteristics of AQP5, its overexpression can have oncogenic effects $(11,21,23)$, including in cancer of the ovary (24), cervix (25) and colon (26), as well as in ovarian epithelium (27) and gastric carcinoma cells (28). However, in lung cancer, it remains to be determined whether the silencing of AQP5 expression affects lung cancer development and the underlying mechanisms remain to be elucidated. In the present study, the effects of AQP5 silencing on the proliferation, cell cycle progression and apoptosis of A549 lung cancer cells through the regulation of ERK signaling, as well as on the promotion of apoptosis in lung cancer xenograft tumors in nude mice, were investigated. It was observed that silencing of AQP5 inhibited the proliferation and promoted the apoptosis 
of A549 cells in vitro and in vivo, suggesting that it may constitute a promising target in the development of novel drugs for lung cancer treatment.

Proliferation, cell cycle progression and apoptosis are the major mechanisms responsible for cell growth. Extension of the G1/S phase transition can effectively inhibit cell proliferation (29). The results of the present study demonstrated that AQP5 was highly expressed in A549 lung cancer cells and functioned as a negative regulator of cell cycle progression. Silencing of AQP5 in A549 cells by RNA interference resulted in cell cycle arrest, with an accumulation of cells in G0/G1 phase and reduced numbers of cells in $\mathrm{S}$ and $\mathrm{G} 2 / \mathrm{M}$ phases observed. Apoptosis is a well-orchestrated cellular mechanism that helps maintain the balance between cell proliferation and death, the disruption of which is considered to be an early and important event in cancer (30). Apoptosis is regulated by multiple genes at the cellular level, including cleaved caspase-3, Bcl-2 and Bax. Caspase-3 is an effector caspase that initiates cell degradation in the final stages of apoptosis. In addition, Bax, a pro-apoptotic protein, and Bcl-2, a survival-promoting protein, are members of the Bcl-2 family that serve key roles in the regulation of intrinsic apoptotic signaling (31-33). The current study revealed that silencing of AQP5 expression in A549 cells effectively promoted non-viable apoptotic cell increase in vitro and in vivo, downregulating the expression of $\mathrm{Bcl}-2$, and elevating the levels of Bax and cleaved caspase-3.

The ERK1/2 pathway is a classic mitogen-activated protein kinase signaling cascade that regulates cell proliferation and growth $(34,35)$. In the present study, it was observed that silencing of AQP5 inhibited ERK1/2 signaling, consistent with the observations of prior studies $(17,36)$. The downstream ERK signaling proteins c-Fos and CREB, which are present in the eukaryotic nucleus, are phosphorylated upon the activation of ERK signaling (37). The current study results also demonstrated that AQP5 silencing reduced the protein levels of c-Fos and p-CREB. These results suggest that AQP5 downregulation may exert an inhibitory effect on A549 lung cancer cell growth by reducing ERK1/2 signaling.

In conclusion, the present study demonstrated that AQP5 silencing inhibited the proliferation and cell cycle progression of A549 lung cancer cells, and that it inducted induce cell apoptosis in vitro and in vivo. Furthermore, this inhibitory effect may be brought about by restriction of ERK1/2 signaling pathway activation. Nevertheless, as AQP5 is widely expressed, further studies of the mechanisms responsible for these effects should be conducted to assess its potential application as a therapeutic target.

\section{Acknowledgements}

Not applicable.

\section{Funding}

This study was supported by the Jilin Province major scientific and technological projects (grant no. 20160201001YY) .

\section{Availability of data and materials}

Not applicable

\section{Authors' contributions}

GZ was the overall advisor of the study; she formulated the experiment plan and examined the accuracy of the experimental results; $\mathrm{LZ}$ was involved in the performing of the experiments; JL, HZ and ZD provided experimental and operational support. All authors have read and approved the final manuscript.

\section{Ethics approval and consent to participate}

Animal care and treatment and all animal experiments were approved by the Experimental Animal Ethics Committee of Jilin University (Changchun, China).

\section{Consent for publication}

Not applicable.

\section{Competing interests}

The authors declare that they have no competing interests.

\section{References}

1. Chen W, Zheng R, Baade PD, Zhang S, Zeng H, Bray F, Jemal A, Yu XQ and He J: Cancer statistics in China, 2015. CA Cancer J Clin 66: 115-132, 2016.

2. Siegel RL, Miller KD and Jemal A: Cancer statistics, 2016. CA Cancer J Clin 66: 7-30, 2016.

3. Jin C, Zhang G, Zhang Y, Hua P, Song G, Sun M, Li X, Tong T, $\mathrm{Li} \mathrm{B}$ and Zhang $\mathrm{X}$ : Isoalantolactone induces intrinsic apoptosis through p53 signaling pathway in human lung squamous carcinoma cells. PLoS One 12: e0181731, 2017.

4. He F, Du T, Jiang Q and Zhang Y: Synergistic effect of Notch-3specific inhibition and paclitaxel in non-small cell lung cancer (NSCLC) cells via activation of the intrinsic apoptosis pathway. Med Sci Monit 23: 3760-3769, 2017.

5. Ridge CA, McErlean AM and Ginsberg MS: Epidemiology of lung cancer. Semin Intervent Radiol 30: 93-98, 2013.

6. Wang L, Li R, Che K, Liu Z, Xiang S, Li M and Yu Y: Enhanced antitumor effect on intrapulmonary tumors of docetaxel lungtargeted liposomes in a rabbit model of VX2 orthotopic lung cancer. Sci Rep 7: 10069, 2017.

7. Howington JA, Blum MG, Chang AC, Balekian AA and Murthy SC: Treatment of stage I and II non-small cell lung cancer: Diagnosis and management of lung cancer, 3rd ed: American College of Chest Physicians evidence-based clinical practice guidelines. Chest 143: e278S-e313S, 2013.

8. Collisson EA, Campbell JD, Brooks AN, Berger AH, Lee W, Chmielecki J, Beer DG, Cope L, Creighton CJ, Danilova L, et al; Cancer Genome Atlas Research Network: Comprehensive molecular profiling of lung adenocarcinoma. Nature 511: 543-550, 2014.

9. Wang M, Lin T, Wang Y, Gao S, Yang Z, Hong X and Chen G: CXCL12 suppresses cisplatin-induced apoptosis through activation of JAK2/STAT3 signaling in human non-small-cell lung cancer cells. Onco Targets Ther 10: 3215-3224, 2017.

10. Agre P and Kozono D: Aquaporin water channels: Molecular mechanisms for human diseases. FEBS Lett 555: 72-78, 2003.

11. Shen Q, Lin W, Luo H, Zhao C, Cheng H, Jiang W and Zhu X: Differential expression of aquaporins in cervical precursor lesions and invasive cervical cancer. Reprod Sci 23: 1551-1558, 2016.

12. Agre P: The aquaporin water channels. Proc Am Thorac Soc 3: 5-13, 2006.

13. Verkman AS: More than just water channels: Unexpected cellular roles of aquaporins. J Cell Sci 118: 3225-3232, 2005.

14. Chae YK, Woo J, Kim MJ, Kang SK, Kim MS, Lee J, Lee SK, Gong G, Kim YH, Soria JC, et al: Expression of aquaporin 5 (AQP5) promotes tumor invasion in human non small cell lung cancer. PLoS One 3: e2162, 2008. 
15. Jung HJ, Park JY, Jeon HS and Kwon TH: Aquaporin-5: A marker protein for proliferation and migration of human breast cancer cells. PLoS One 6: e28492, 2011.

16. Lee SJ, Kang BW, Kim JG, Jung JH, Lee J, Kim WW, Park HY, Jeong JH, Jeong JY, Park JY, et al: AQP5 variants affect tumora expression of AQP5 and survival in patients with early breast cancer. Oncology 92: 153-160, 2017.

17. Yang J,Zhang JN, Chen WL, Wang GS, Mao Q,LiSQ, Xiong WH, Lin YY, Ge JW, Li XX, et al: Effects of AQP5 gene silencing on proliferation, migration and apoptosis of human glioma cells through regulating EGFR/ERK/ p38 MAPK signaling pathway. Oncotarget 8: 38444-38455, 2017.

18. Xin LB, Jiang XX, Ye XL, Wu RJ, Xu KH, Ma JY and Lin J: AQP5 gene silencing inhibits proliferation and migration of ectopic endometrial glandular epithelial cells in endometriosis. Zhejiang Da Xue Xue Bao Yi Xue Ban 44: 285-292, 2015 (In Chinese).

19. Sugihara I, Yoshida M, Shigenobu T, Takagi H, Maruyama K, Takeuchi N, Toda M, Inoue $M$ and Nakada H: Different progression of tumor xenografts between mucin-producing and mucin-non-producing mammary adenocarcinoma-bearing mice. Cancer Res 66: 6175-6182, 2006.

20. Guo K and Jin F: NFAT5 promotes proliferation and migration of lung adenocarcinoma cells in part through regulating AQP5 expression. Biochem Biophys Res Commun 465: 644-649, 2015.

21. Direito I, Madeira A, Brito MA and Soveral G: Aquaporin-5: From structure to function and dysfunction in cancer. Cell Mol Life Sci 73: 1623-1640, 2016.

22. Livak KJ and Schmittgen TD: Analysis of relative gene expression data using real-time quantitative PCR and the 2(-Delta Delta C(T)) Method. Methods 25: 402-408, 2001.

23. Verkman AS, Hara-Chikuma $M$ and Papadopoulos MC: Aquaporins - new players in cancer biology. J Mol Med (Berl) 86: 523-529, 2008.

24. Yang JH, Shi YF, Cheng Q and Deng L: Expression and localization of aquaporin-5 in the epithelial ovarian tumors. Gynecol Oncol 100: 294-299, 2006.

25. Zhang T, Zhao C, Chen D and Zhou Z: Overexpression of AQP5 in cervical cancer: Correlation with clinicopathological features and prognosis. Med Oncol 29: 1998-2004, 2012.

26. Shi X, Wu S, Yang Y, Tang L, Wang Y, Dong J, Lü B, Jiang G and Zhao W: AQP5 silencing suppresses p38 MAPK signaling and improves drug resistance in colon cancer cells. Tumour Biol 35: 7035-7045, 2014.
27. Yan C, Zhu Y, Zhang X, Chen X, Zheng W and Yang J: Downregulated aquaporin 5 inhibits proliferation and migration of human epithelial ovarian cancer $3 \mathrm{AO}$ cells. J Ovarian Res 7: 78, 2014.

28. Huang YH, Zhou XY, Wang HM, Xu H, Chen J and Lv NH: Aquaporin 5 promotes the proliferation and migration of human gastric carcinoma cells. Tumour Biol 34: 1743-1751, 2013.

29. Simpson DJ, Fryer AA, Grossman AB, Wass JA, Pfeifer M, Kros JM, Clayton RN and Farrell WE: Cyclin D1 (CCND1) genotype is associated with tumour grade in sporadic pituitary adenomas. Carcinogenesis 22: 1801-1807, 2001.

30. Qi S, Song Y, Peng Y, Wang H, Long H, Yu X, Li Z, Fang L, Wu A, Luo W, et al: ZEB2 mediates multiple pathways regulating cell proliferation, migration, invasion, and apoptosis in glioma. PLoS One 7: e38842, 2012.

31. Zarnescu O, Brehar FM, Chivu M and Ciurea AV: Immunohistochemical localization of caspase-3, caspase-9 and Bax in U87 glioblastoma xenografts. J Mol Histol 39: 561-569, 2008.

32. Bhattacharjee M, Acharya S, Ghosh A, Sarkar P, Chatterjee S, Kumar P and Chaudhuri S: Bax and Bid act in synergy to bring about T11TS-mediated glioma apoptosis via the release of mitochondrial cytochrome $\mathrm{c}$ and subsequent caspase activation. Int Immunol 20: 1489-1505, 2008.

33. Guo Z, Chen Q, Liu B, Tian D, Zhang S and Li M: LRIG1 enhances chemosensitivity by modulating BCL-2 expression and receptor tyrosine kinase signaling in glioma cells. Yonsei Med J 55: 1196-1205, 2014.

34. McCubrey JA, Steelman LS, Chappell WH, Abrams SL, Wong EW, Chang F, Lehmann B, Terrian DM, Milella M, Tafuri A, et al: Roles of the Raf/MEK/ERK pathway in cell growth, malignant transformation and drug resistance. Biochim Biophys Acta 1773: 1263-1284, 2007.

35. Oh SE and Mouradian MM: Cytoprotective mechanisms of DJ-1 against oxidative stress through modulating ERK1/2 and ASK1 signal transduction. Redox Biol 14: 211-217, 2018.

36. Zhang Z, Chen Z, Song Y, Zhang P, Hu J and Bai C: Expression of aquaporin 5 increases proliferation and metastasis potential of lung cancer. J Pathol 221: 210-220, 2010.

37. Niu C, Xiao F, Yuan K, Hu X, Lin W, Ma R, Zhang X and Huang Z: Nardosinone suppresses RANKL-induced osteoclastogenesis and attenuates lipopolysaccharide-induced alveolar bone resorption. Front Pharmacol 8: 626, 2017. 